\title{
A new characterization of commutative semiregular rings
}

\section{Une nouvelle caractérisation des anneaux semi-réguliers commutatifs}

\author{
Peter Danchev ${ }^{1}$ and Mahdi Samiei ${ }^{2}$ \\ ${ }^{1}$ Institute of Mathematics and Informatics, Bulgarian Academy of Sciences, Sofia, Bulgaria \\ danchev@math.bas.bg;pvdanchev@yahoo.com \\ ${ }^{2}$ Department of Mathematics, Velayat University, Iranshahr, Iran \\ m.samiei@velayat.ac.ir
}

ABSTRACT. A commutative ring $R$ is called $J$-rad clean in case, for any $r \in R$, there is an idempotent $e \in R$ such that $r-e \in U(R)$ and $r e \in J(R)$, where $U(R)$ and $J(R)$ denote the set of units and the Jacobson radical of $R$, respectively. Also, a ring $\mathrm{R}$ is called semiregular if $R / J(R)$ is regular in the sense of von Neumann and idempotents lift modulo $J(R)$. We demonstrate that these two concepts are, actually, equivalent and we portray a portion of the properties of this class of rings. In particular, as a direct application, we prove that the commutative group ring $R G$ is $J$-rad clean if, and only if, $R$ is a commutative $J$-rad clean ring and $G$ is a torsion abelian group, provided that $J(R)$ is nil.

AMS Subject Classification 2010. Primary 16S34, 16U60; Secondary $20 \mathrm{C07.}$

KEYWORDS. Semiregular rings, $J$-clean rings, $J$-rad clean rings.

\section{Introduction and Preliminaries}

The class of strongly rad clean rings were introduced and presented in detail in [12] and some basic properties of these rings have been examined in [8] and [22]. A ring $R$ is called strongly rad-clean in case, for any $r \in R$, there is an idempotent $e \in R$ such that $r-e \in U(R)$, er $=r e$ and $r e \in J(R)$. These rings form a proper subclass of the class of clean rings (see [20] for more information).

A ring $R$ is said to be regular (in the sense of von Neumann) provided that, for any $a \in R$, there exists an element $b \in R$ such that $a=a b a$; in particular, if $b=1$, the ring $R$ is termed boolean. Likewise, a ring $R$ is said to be semiregular if $R / J(R)$ is regular and all idempotents lift modulo $J(R)$ (see, e.g., [19]). In [7], as common applications, semiregularity of Morita contexts and trivial extensions have been investigated. Some characterizations of rings $R$ have been given such that idempotents lift modulo $J(R)$, and $R / J(R)$ satisfies one of the following conditions: (one-sided) unit-regular, strongly regular, (unit, strongly, weakly) $\pi$-regular, etc. ([4]).

Throughout the present paper, all given rings are thought to be commutative with nonzero identity and all given groups are thought to be multiplicative abelian. The set of units and idempotents of $R$ shall be denoted by $U(R)$ and $I d(R)$, respectively. As usual, $J(R)$ stands for the Jacobson radical of $R$ and $N(R)$ for the nil-radical of $R$. Our basic notations as well as all further settings and the terminology are in agreement with [16].

We recall that a ring $R$ is $J$-rad clean, provided that, for any $r \in R$, there exists an idempotent $e \in R$ and a unit $u \in R$ such that $r=e+u$ and $r e \in J(R)$. These rings are, actually, the commutative variant of the aforementioned strongly rad-clean rings (see, for more account, [8] too).

Our motivation to write up this article is to describe $J$-rad clean rings and also to demonstrate the curious fact that the class of $J$-rad clean rings are actually the class of semiregular rings. 
Before presenting the main results quoted below in detail, we need some background material for completeness of the exposition and for the reader's convenience. In that aspect, the next two chief concepts can be found in [9] and [8], respectively.

Definition 1.1. A ring $R$ is called $J$-clean if, for every $r \in R$, there exists an idempotent $e \in R$ such that $r-e \in J(R)$.

It follows from results in [9] as well as by a direct inspection that a ring $R$ is $J$-clean $\Longleftrightarrow$ the factor-ring $R / J(R)$ is boolean and idempotents lift modulo $J(R)$.

Our key instrument here is the following concept.

Definition 1.2. A ring $R$ is called $J$-rad clean if, for every $r \in R$, there exists an idempotent $e \in R$ such that $r-e \in U(R)$ and $r e \in J(R)$.

We begin our work with the following simple but useful technical claim, which observation will be freely used in the sequel (compare also with [8]).

Lemma 1.3. If $R$ is a $J$-rad clean ring with $J(R)=0$, then $R$ is regular.

Proof. For any element $r \in R$, we write that $r=u+e$ where $u \in U(R), e \in I d(R)$ with $r e=0$. Thus $e u=u e=-e$ and $u^{-1} e=e u^{-1}=-e$. Furthermore, one observes that $r u^{-1} r=(u+e) u^{-1}(u+e)=$ $(1-e)(u+e)=(1-e) u=u+e=r$. This constatation means that $R$ is surely regular, as claimed.

Proposition 1.4. The following statements are true:

(1) The class of J-rad clean rings is closed under homomorphic images. In addition, $R$ is a $J$-rad clean ring if and only if $R / J(R)$ is a regular ring and idempotents lift modulo $J(R)$. In particular, $R$ is a J-rad clean ring if and only if the quotient $R / I$ is a J-rad clean ring, where $I \subseteq J(R)$, and idempotents lift modulo $J(R)$.

(2) A regular ring $R$ is $J$-rad clean.

(3) A $\pi$-regular ring (that is, a zero-dimensional ring) is J-rad clean.

Proof. (1). It is rather straightforward to prove this point directly, so we omit the details by leaving them to the interested reader for a direct check. However, we will sketch the proof more conceptually. The necessity follows by usage of Lemma 1.3. As for the sufficiency, the map $R / I \rightarrow R / J(R)$ is an epimorphism with the kernel $J(R) / I$. Hence $R / J(R)$ is a $J$-rad clean ring and so it is regular utilizing Lemma 1.3, We now may apply [8, Corollary 2.2].

(2). Suppose that $r$ is an element of regular ring $R$. Then, by the utilization of [2, Theorem 2.2], we know that $r=u e$ for some unit element $u$ and an idempotent element $e$ in $R$. Now, by taking $v=u e-(1-e)$ and $f=1-e$, it follows then that $r=v+f$ with $r f \in J(R), v \in U(R)$ with the inverse $v^{-1}=e u^{-1}-(1-e)$ and $f \in \operatorname{Id}(R)$.

(3). Let $R$ be a zero-dimensional ring. Then, it is simple verified that $R / N(R)$ is a zero-dimensional reduced ring, and so it is a regular ring. It follows from part (2) that $R / N(R)$ is $J$-rad clean and hence $R$ is $J$-rad clean by utilizing part (1) as $J(R)$ is always nil (i.e., $J(R)=N(R)$ ). 
The next two characterization results are pivotal. Recall that a ring is said to be indecomposable, provided all its idempotents are only the trivial ones, namely 0 and 1 .

Theorem 1.5. The following are equivalent for a ring $R$ :

(1) $R$ is an indecomposable J-rad clean ring.

(2) $R$ is a local ring.

(3) $J(R)$ is prime and $R$ is a $J$-rad clean ring.

(4) $R$ is an indecomposable clean ring.

(5) $R=U(R) \cup J(R)$.

Proof. $(1) \Rightarrow(2)$ is clear by definition.

$(2) \Rightarrow(1)$. Suppose that $(R, M)$ is a local ring. If $r \in U(R), r=r+0$. Otherwise, $r \in M$ and so $r-1 \in U(R)$ and hence $r=(r-1)+1$. Thus $R$ is an indecomposable $J$-rad clean.

$(2) \Rightarrow(3)$ is clear by using similar method of the proof $(2) \Rightarrow(1)$.

$(3) \Rightarrow(2)$. Assume that $R$ is a $J$-rad clean ring. For any $r \in R$, we can write $r=u+e$ such that $r e \in J(R)$ where $u \in U(R)$ and $e \in I d(R)$. Since $J(R)$ is prime, we have $r \in J(R)$ or $e \in J(R) \cap I d(R)$. Thus $R=J(R) \cup U(R)$ and so $R$ is a local ring.

(2) $\Leftrightarrow(4)$ is true by [1, Theorem 3].

(1) $\Leftrightarrow(5)$ is clearly true.

So we come to our chief theorem.

Theorem 1.6. The following statements are equivalent for a ring $R$ :

(1) $R / J(R)$ is regular and every idempotent lifts modulo $J(R)$.

(2) For any $x \in R$, there exist an idempotent $e \in R$ and a unit $u \in R$ such that $\bar{x}=\overline{e u}$ in $R / J(R)$.

(3) For any $x \in R$, there exist an idempotent $e \in R$, a unit $u \in R$ and $j \in J(R)$ such that $x=j+e u$.

(4) $R$ is J-rad clean.

(5) $R / J(R)$ is $J$-rad clean and every idempotent lifts modulo $J(R)$.

(6) For any $x \in R$, there exist a unit $u$ and an idempotent $e$ in $R x$ such that $x-e u \in J(R)$.

(7) For any $x \in R$, there exist $a$ unit $u$ and an idempotent $e \in R x$ such that $(1-e) x \in J(R)$ and $u x$ is an idempotent of $R / J(R)$.

(8) For every element $x$ in $R$ there exists an element $b$ in $R$ such that $(x b)^{2}=x b$ and $x-x^{2} b \in J(R)$.

Proof. $(1) \Rightarrow(2)$. For any $x \in R$, there exists an element $y \in R$ such that $\bar{x}=\overline{x^{2} y}$ in $R / J(R)$. Set $z=y^{2} x$. Then $\overline{x^{2} z}=\overline{x^{3} y^{2}}=\bar{x}$ and so $\bar{z}=\overline{y^{2} x^{2} z}=\overline{z^{2} x}$. Set $u=x+1-x z$. It is easy to verify that

$$
\overline{(z+1-x z) u}=\overline{1}
$$


Hence, $u \in U(R)$. Let $e=x z$. Then we can find an idempotent $f \in R$ such that $\bar{e}=\bar{f}$. In addition, $\bar{x}=\overline{f u}$.

(2) $\Rightarrow(3)$ is easy.

(3) $\Rightarrow(4)$. For any $x \in R$, there exist an idempotent $e \in R, j \in J(R)$ and a unit $u \in R$ such that $x=j+e u$. Let $f=1-e$ and $w=x-f$. Choose $v=u^{-1} e-f$. Then $w v=(x-f)\left(u^{-1} e-f\right)=x u^{-1} e+(1-x) f-f u^{-1} e$ and so in $\bar{R}=R / J(R)$, we have $\overline{w v}=\overline{e u} \overline{u^{-1}} \bar{e}+(\overline{1}-\overline{e u}) \bar{f}-\overline{f u^{-1} e}=\overline{e+f}=\overline{1}$. Thus $\bar{w} \in U(\bar{R})$ and so $w \in U(R)$. Therefore, $x=f+w$ is a sum of an idempotent $f \in R$ and a unit $w \in R$. One easily checks that $f x \in J(R)$, as required.

(4) $\Rightarrow(5)$. By Proposition $1.4, \bar{R}=R / J(R)$ is $J$-rad clean since $R$ is $J$-rad clean. Also, in view of the main result in [20], each idempotent lifts modulo $J(R)$.

$(5) \Rightarrow(1)$. Suppose that $R / J(R)$ is $J$-rad clean and idempotent lifts modulo $J(R)$. Thus, for any $\bar{r} \in \bar{R}$ we can find $e \in I d(R)$ such that $\bar{r}-\bar{e} \in U(\bar{R})$ and $\bar{r} \bar{e}=\overline{0}$. It follows from [2, Theorem 2.2(5)] that $R / J(R)$ is regular, as required.

(1) $\Leftrightarrow(6) \Leftrightarrow(7)$ by [4, Theorem 2.14].

(1) $\Leftrightarrow(8)$ by [21, Proposition 1].

Remark. To keep the record straight, we just notice that the equivalence (2) $\Longleftrightarrow$ (4) was also proved in [8, Theorem 2.1] and the equivalence (1) $\Longleftrightarrow(4)$ was also showed in [8, Corollary 2.2] (let us note also that it was directly obtained in Proposition 1.4 listed above).

The next technicality is important.

Lemma 1.7. [16. Exercise 19A, page 270] Let $R$ be a ring. For any $a \in R$, the following are equivalent:

(1) $a=a^{2} v$ for some $v \in U(R)$.

(2) $a=$ eu for some $e \in I d(R)$ and $u \in U(R)$.

(3) $a=a^{2}$ sfor some $s \in R$, and $R / a R \cong \operatorname{ann}(a)$ as $R$-modules.

(4) $R a$ is a direct summand of $R$, and $R / a R \cong$ ann(a) as $R$-modules.

A ring $R$ in which its elements satisfy the equivalent conditions in Lemma 1.7 is called a unit-regular ring. Likewise, [2, Theorem 2.2] shows that the two concepts of commutative unit-regular rings and commutative regular rings are the same.

The following assertion is useful.

Theorem 1.8. [5] Theorem 1] The following are equivalent for a ring $R$ :

(1) $R / J(R)$ is unit-regular.

(2) Every element $\bar{a}$ of $\bar{R}=R / J(R)$ can be written as $\bar{e}+\bar{u}$ such that $\bar{R} \bar{a} \cap \bar{R} \bar{e}=\overline{0}$, where $\bar{e}$ is an idempotent and $\bar{u}$ a unit in $\bar{R}$.

On the other side, in [6], a ring $R$ is called semi unit-regular if idempotents lift modulo $J(R)$ and $R / J(R)$ is unit-regular. Therefore, the three concepts semi unit-regular, semiregular and $J$-rad clean rings are the same by virtue of Theorem 1.6 .

The following is an example of a clean ring that is not $J$-rad clean. 
Example 1.9. [3, Example 2.7]

Let $\mathbb{Q}$ be the field of rational numbers and $L$ the ring of all rational numbers with odd denominators. Define

$$
R=\left\{\left(r_{1}, \cdots r_{n}, s, s, \cdots\right) \mid n \geq 1, r_{1}, \cdots, r_{n} \in \mathbb{Q}, s \in L\right\} .
$$

It is easy to check that $\mathrm{R}$ is a commutative clean ring, while it is not $J$-rad clean.

Recall that $R$ is a $\pi$-regular ring if, for every $a \in R$, there are $x \in R$ and an integer $n \geq 1$ such that $a^{2 n} x=a^{n}$. It is well known that these rings are exactly the zero-dimensional ones.

The following statement is trivial, but we state it here only for completeness.

Corollary 1.10. Let $R$ be a ring. The following are equivalent:

(1) $R$ is $\pi$-regular.

(2) $R$ is zero-dimensional.

(3) $R / N(R)$ is regular.

(4) $J(R)$ is nil (i.e., $J(R)=N(R)$ ) and $R$ is $J$-rad clean.

Proof. (1) $\Leftrightarrow(2) \Leftrightarrow(3)$ are well-known by [13, Theorem 3.1, page 10].

$(2) \Rightarrow(4)$ is true by Proposition $1.4(3)$.

(4) $\Rightarrow(3)$ is clear by Theorem 1.6 .

An other result of interest is the following one.

Theorem 1.11. Let $R$ be a ring. Then $R$ is $J$-rad clean with $U(R)=1+J(R)$ (thus, in particular, $2 \in J(R)$ ) if and only if $R$ is $J$-clean.

Proof. For the sufficiency, since $R$ is a $J$-clean ring, one writes that $2=x+e$ for some $x \in J(R)$ and $e \in I d(R)$. Therefore, $1-e=x-1 \in I d(R) \cap U(R)$. Thus $x-1=1$ and so $x=2 \in J(R)$ - notice also that this fact follows automatically from the condition $U(R)=1+J(R)$ because $-1 \in U(R)$. To prove $R$ is $J$-rad clean, observe that for any $r \in R$, we have $r-1=y+e$ for some $y \in J(R)$ and $e \in I d(R)$. So, $r=1+y+e$ such that $1+y \in U(R)$ and $e \in I d(R)$. Now, $r e=(2+y) e \in J(R)$ since $2 \in J(R)$. Therefore, $R$ is $J$-rad clean. The remaining part-half that $U(R)$ contains only units from $1+J(R)$ is pretty evident as $U(R)+J(R)=U(R)$.

Conversely, suppose that $R$ is $J$-rad clean and $U(R)=1+J(R)$. As we have seen, 2 belongs to $J(R)$, so we may assume that $2=0$ in the quotient $R / J(R)$, and thus $\operatorname{char}(R / J(R))=2$. Since $U(R / J(R))$ is trivial, it is therefore obvious with the aid of Theorem 1.6(1) that $R / J(R)$ is a boolean ring and so by what we have commented above one finds that the ring $R$ is surely $J$-clean, as promised.

By considering the four elements field $\mathbb{F}_{4}$ of characteristic 2 , one detects that the requirement on the units of $R$ cannot be ignored and thereby the condition $2 \in J(R)$ is definitely not enough to extract the wanted equivalence.

The next two resulting consequences of the previous assertion are immediate, but we will give some independent proofs which could be helpful (compare with [9] as well). 
Corollary 1.12. Consider the following statements for a ring $R$ :

(1) $R$ is J-rad clean.

(2) $R$ is clean.

(3) $R$ is J-clean.

Then (1) $\Rightarrow(2)$ and $(3) \Rightarrow(2)$. Moreover, if $R$ is $J U$, that is $U(R)=1+J(R)$, then the above three statements are equivalent.

Proof. The first two claimed implications are pretty obvious, so we are concentrated on the second part when $R$ is a JU-ring. (2) $\Rightarrow(1)$. Then, by virtue of [9] or [11], the quotient $R / J(R)$ is a boolean ring and idempotents lift modulo $J(R)$. Thus, we conclude from Theorem 1.6 that $R$ is $J$-rad clean (and even $J$-clean).

$(2) \Rightarrow(3)$ is clear by [9] or [11] or we may argue as in the preceding implication.

Corollary 1.13. Let $R$ be a ring. Consider the following statements:

(1) $R / J(R)$ is a boolean ring.

(2) $U(R / J(R))=\{1\}$.

(3) $\operatorname{char}(R / J(R))=2$.

(4) $R$ is a JU-ring.

(5) $R$ is a J-clean ring.

(6) $2 \in J(R)$.

Then, (1) $\Rightarrow$ (2) $\Leftrightarrow$ (4), (1) $\Rightarrow$ (3), (5) $\Rightarrow$ (6) and (5) $\Rightarrow$ (1). Moreover, if R is J-rad clean with $U(R)=1+J(R)$, then the above six statements are equivalent.

Proof. (1) $\Rightarrow(2),(1) \Rightarrow$ (3) and (5) $\Rightarrow$ (1) are trivially true.

$(2) \Leftrightarrow(4)$ is true by [9] or [11].

$(5) \Rightarrow(6)$ is true by Theorem 1.11 .

Now, suppose that $R$ is simultaneously a JU-ring and a $J$-rad clean ring.

$(2) \Rightarrow(1)$. Let $R$ be a $J$-rad clean ring such that $U(R / J(R))=\{1\}$. By Theorem 1.6(2) for any $r \in R$, we know that $\bar{r}=\overline{e u}$ for some $u \in U(R)$ and $e \in I d(R)$ and hence $R / J(R)$ is boolean.

(3) $\Rightarrow(1)$. Suppose that $R$ is a $J$-rad clean ring and $\operatorname{char}(R / J(R))=2$. By application of Theorem 1.11, $R$ is a $J$-clean ring, and so the factor-ring $R / J(R)$ is a boolean ring, as desirable.

(4) $\Rightarrow$ (5). By Theorem 1.6(3), for any $r \in R$ we can write $r=j+e u$ for some $e \in I d(R), u \in U(R)$ and $j \in J(R)$. Since $R$ is JU, there is $k \in J(R)$ such that $u=1+k$ and so $r=l+e$ where $l=j+e k \in J(R)$. Thus $R$ is $\mathrm{J}$-clean.

$(6) \Rightarrow(5)$ is clear by Theorem 1.11 .

Recall that a ring $R$ is called semiperfect if idempotents lift modulo $J(R)$ and $R / J(R)$ is artinian. Semiperfect rings are known to be semiregular as well as it is easy to see that Theorem 1.6 implies that a semiperfect ring is also $J$-rad clean.

Theorem 1.14. For a ring $R$, the following statements are equivalents: 
(1) $R$ is semiperfect.

(2) $R$ is J-rad clean and contains no infinite family of orthogonal idempotents.

(3) $R$ is clean and contains no infinite family of orthogonal idempotents.

The next statement gives only one way conditions on the $\operatorname{ring} R$ and the group $G$ under which the group ring $R G$ is clean. So far there is no a complete answer only in terms of $R$ and $G$ and their sections when $R G$ is clean.

Lemma 1.15. ([18. Proposition 2.7];[14, Proposition 4.8]) The coefficient ring $R$ must be clean and the underlying group $G$ must be torsion in order for the commutative group ring $R G$ to be clean.

The following result sheds some more light on the question of when the group ring $R G$ is $J$-rad clean under the additional assumption that $J(R)$ must be nil (that is, $J(R)=N(R)$ ).

Theorem 1.16. The following are equivalent for a ring $R$ with nil $J(R)$ and a group $G$ :

(1) $R G$ is a commutative $J$-rad clean ring.

(2) $R$ is a $J$-rad clean ring and $G$ is a torsion abelian group.

Proof. (1) $\Rightarrow$ (2). Suppose that $R G$ is a $J$-rad clean ring. Considering the standard augmentation map $R G \rightarrow R$, one finds that $R$ is $J$-rad clean, by Proposition 1.4(1). Also, by definition, $R G$ is clean and so $G$ is torsion by Lemma 1.15 .

$(2) \Rightarrow(1)$. Let $R$ be a $J$-rad clean ring with $J(R)=N(R)$. Then, with Theorem $1.6(1)$ at hand, we get that $R / J(R)$ is regular and hence $R G / J(R G)$ is regular since $(R / J(R)) G \cong R G /(J(R) G)$ and $\frac{R G /(J(R) G)}{J(R G) /(J(R) G)} \cong$ $R G / J(R G)$ - see, e.g., [21] and [10]. To complete the proof, by Theorem $1.6(1) \Rightarrow(4)$, it remains to show that every idempotent lift modulo $J(R G)$. To that aim, the utilization of the corresponding results from [15] and [17], respectively, lead us to the fact that $J(R G)=N(R G)$, i.e. that $J(R G)$ is nil, as required.

Acknowledgements. The authors would like to thank the anonymous referee for his/her insightful comments and suggestions. The work of the first named author P. V. Danchev is partially supported by the Bulgarian National Science Fund under Grant KP-06 N 32/1 of Dec. 07, 2019.

\section{References}

[1] D.D. Anderson and V.P. Camillo, Commutative rings whose elements are a sum of a unit and an idempotent, Commun. Algebra 30 (2002), 3327-3336.

[2] D.F. Anderson and A. Badawi, Von Neumann regular and related elements in commutative rings, Algebra Colloq. 19 (Spec 1) (2012) 1017-1040.

[3] N. Ashrafi and E. Nasibi, Rings in which elements are the sum of an idempotent and a regular element, Bull. Iran Math. Soc. 39(3) (2013), 579-588.

[4] P. Aydog du, Y. Lee and A. C. ÖZcan, Rings close to semiregular, J. Korean Math. Soc. 49(3) (2012), 605-622.

[5] V.P. Camillo and D. Khurana, A characterization of unit regular rings, Commun. Algebra 29(5) (2001), 2293-2295.

[6] V.P. Camillo and H.-P. Yu, Exchange rings, units and idempotents, Commun. Algebra 22 (1994), 4737-4749. 
[7] H. Chen and M. Chen, On semiregular rings, New Zealand J. Math. 32 (2003), 11-20.

[8] H. Chen, Some classes of strongly clean rings, Bull. Iran. Math. Soc. 39(6) (2013), 1099-1115.

[9] P.V. Danchev, Rings with Jacobson units, Toyama Math. J. 38 (2016), 61-74.

[10] P.V. Danchev, Commutative nil-clean and $\pi$-regular group rings, Uzbek Math. J. (2019)(3), 33-39.

[11] P.V. Danchev, On exchange $\pi-J U$ unital rings, Rend. Sem. Mat. Univ. Pol. Torino 77(1) (2019), 13-23.

[12] A.J. Diesl, Classes of Strongly Clean Rings, Ph.D. Thesis, University of California, Berkeley, 2006.

[13] J.A. Huckaba, Commutative Rings with Zero Divisors, Marcel Dekker, New York-Basel, 1988.

[14] N.A. Immormino, Clean Rings and Clean Group Rings, Ph.D. Thesis, Bowling Green State University, 2006.

[15] G. Karpilovsky, The Jacobson radical of commutative group rings, Arch. Math. (Basel) 39 (1982), 428-430.

[16] T.-Y. Lam, Lectures on Modules and Rings, Graduate Texts in Mathematics, 189, Springer-Verlag, New York, 1999.

[17] W.L. May, Group algebras over finitely generated rings, J. Algebra 38 (1976), 483-511.

[18] W.Wm. McGovern, A characterization of commutative clean rings, Int. J. Math. Game Theory \& Algebra 15 (2006), 403-413.

[19] W.K. Nicholson, Semiregular modules and rings, Can. J. Math. 28 (1976), 1105-1120.

[20] W.K. Nicholson Lifting idempotents and exchange rings Trans. Amer. Math. Soc. 229 (1977), 269-278.

[21] J.B. Srivastava and S.K. Shah, Semilocal and semiregular group rings, Indag. Math. (Proceedings) 83(3) (1980), $347-352$

[22] Z.L. Ying, Clean Rings and Regular Local Rings, Ph.D. Thesis, Southeast University, Nanjing, 2008. 